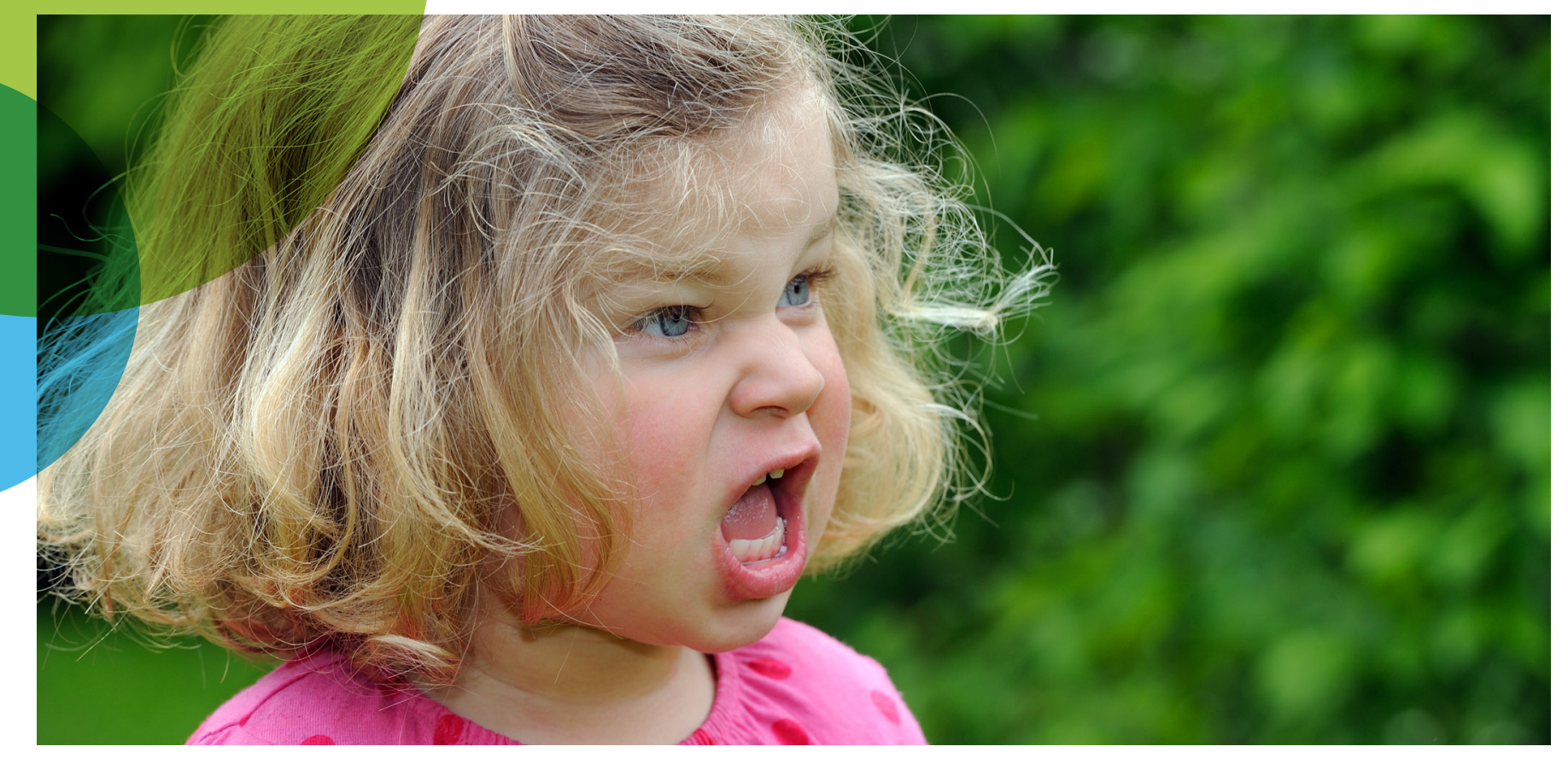

\title{
Aggression toward siblings during the preschool years: when does it become atypical?
}

\author{
By Dr. Melanie Dirks
}

Dr. Dirks is Associate Professor in the Department of Psychology at McGill University, Montréal, Canada. Data for this study came from the Multidimensional Assessment of Preschoolers Study (MAPS), a National Institute of Mental Health study led by Dr. Lauren Wakschlag of Northwestern University.

This article is a summary of the paper published in JCPP - Dirks MA, Recchia HE, Estabrook R, Howe N, Petitclerc A, Burns JL, Briggs-Gowan MJ, Wakschlag LS. (2019). Differentiating Typical from Atypical Perpetration of Sibling-Directed Aggression during the Preschool Years. Journal of Child Psychology and Psychiatry, 60, 267-276. doi.org/10.1111/jcpp.12939

Most children grow up with siblings. During early childhood, siblings spend a great deal of time together and must navigate challenging situations such as sharing toys and parental attention, features that make conflict inevitable and often emotionally intense. Preschool-aged siblings have been observed to fight every 10 minutes, and these conflicts often escalate to include aggressive behaviours, such as hitting, name-calling, and exclusion. Aggression between siblings is so common that it is often viewed as a normative right of passage by parents and clinicians. Indeed, the DSM-5 criteria for oppositional defiant disorder state that children who demonstrate symptoms - including vindictiveness - only with a sibling do not meet diagnostic criteria. However, several recent studies conducted with large and representative samples have made it clear that these behaviours can be harmful for the victims.
Aggression toward siblings may also be a marker of clinical risk for the perpetrator. Given how common sibling-directed aggression is, for most children it will be part of a typical developmental trajectory. For some children, however, these actions may indicate significant behavioural or emotional dysfunction that will escalate with time. It is critical that we identify markers that allow us to differentiate typical and atypical sibling aggression, particularly during the preschool years, when aggressive behaviours are much more modifiable. In some cases, qualitative features of the behaviour may mark it as severe. For example, few preschoolers engage in aggression that is purposefully intended to harm another, and this behaviour is worrisome even if it occurs rarely. Other, more common misbehaviours, such as teasing a brother or sister or excluding them from play, may also be atypical when exhibited very frequently. 
In other words, many preschoolers will taunt a sibling sometimes, but few preschoolers will do it a lot, and those children may be at risk for emotional and behavioral disorders.

In this study, we identified the frequencies at which sibling-directed aggression became atypical; that is, fewer than $5 \%$ of preschoolers were reported to be engaging in the behaviour that often. Data were obtained from the MAPS Study, a NIMH-funded investigation directed by Lauren Wakschlag of Northwestern University. An ethnically and socioeconomically diverse sample of more than 1500 parents of 3- to 5-year-olds reported on their children's aggressive and disruptive behaviour. Typically, researchers ask parents to provide general judgments about how often their children engage in a given behaviour (e.g., "never", "sometimes", or "a lot"). In contrast, we asked parents to provide precise frequency estimates (i.e., "never in the last month," "rarely [less than once per week]," "some [1-3] days of the week," "most [4-6] days of the week," "every day of the week," "many times each day"). Doing so allowed us to generate thresholds of practical concern; that is, exactly how often must a behaviour be occurring before it becomes atypical? In general, we found that aggression toward brothers and sisters - including hitting, shoving or kicking them, saying or doing mean things behind their back, and teasing or taunting them - became atypical when it was occurring most days. In contrast, the same behaviours, when they were directed toward a peer, became atypical when they occurred some days.

Several limitations of our study must be kept in mind when interpreting the results. First, our finding that sibling aggression becomes atypical when it occurs most days is based on parent reports of their children's behaviour. These cut-off points should not be applied to estimates of the frequency of sibling aggression obtained using other types of measures (e.g., observation). Nonetheless, parents are excellent informants of their children's behaviour, and parent-report instruments can be used in many health-care settings, making them an ideal tool for identifying children who are demonstrating clinically concerning behaviours. Second, we did not examine positive features of the sibling relationship, such as warmth between brothers and sisters. It may be that even frequent sibling-directed aggression is less concerning when it occurs in the context of a generally loving relationship. It will be important for future studies to examine this possibility.

These findings are a first step toward specifying features of sibling-directed aggression that may be markers of clinical risk for the aggressor. The critical next step is to determine whether the frequency with which children engage in sibling-directed aggression is linked to subsequent behavioural and

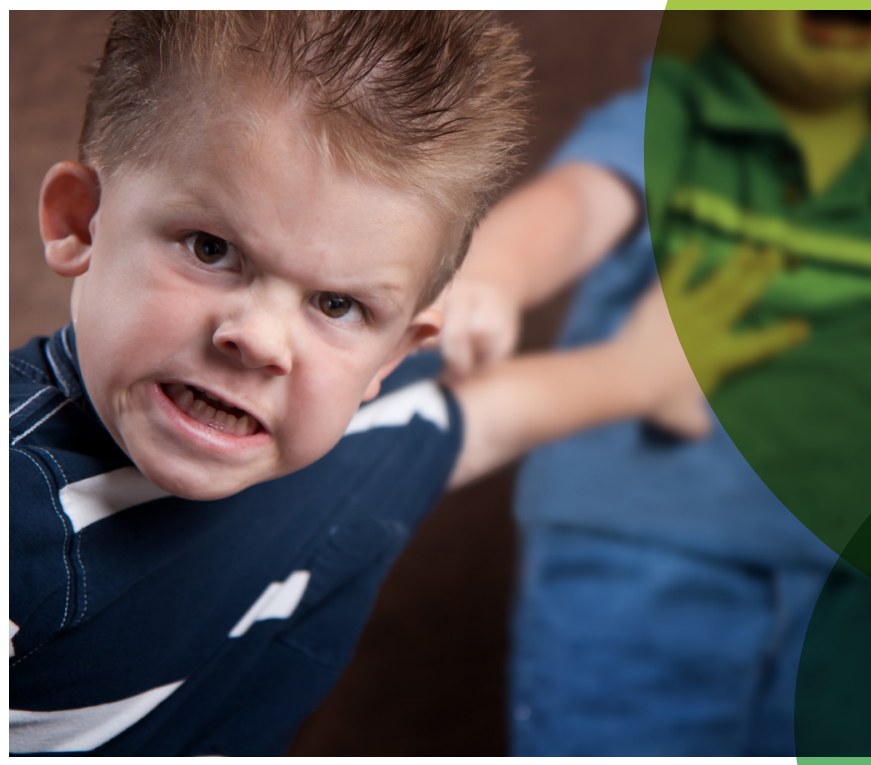

emotional problems. Ultimately, this research may provide empirically-based guidelines that will help practitioners identify children who would benefit from early intervention. Many 3- and 4-year-olds do not attend preschool, which limits opportunities to observe their aggression toward peers. However, parents are well-positioned to report on behaviours occurring between brothers and sisters. In addition, many parents are concerned about fighting and aggression between their children, and as a result, they may be likely to mention these behaviours to health-care practitioners and to seek guidance as to whether these behaviours are clinically concerning.

\section{Key points:}

- Sibling aggression can be harmful for victims; it may also indicate that the perpetrator is experiencing or at risk for emotional and behavioural disorders.

- It is therefore critical to differentiate typical from atypical sibling aggression, particularly during the preschool years, when aggressive behaviour is more modifiable.

- Working with reports from more than 1500 parents of preschoolers, we identified the frequency at which sibling-directed aggression became atypical (i.e., less than $5 \%$ of children were reported to engage in the behaviour that often).

- Most aggressive behaviours directed toward siblings - including hitting, name calling, and excluding a sibling - were atypical when they occurred most days; the same behaviors targeted at peers were atypical when they occurred some days.

- Results are a first step toward identifying features of sibling aggression that may be markers of clinical risk for the aggressor. 